\title{
Cerámica libre de metal terminada y caracterizada por pulido manual
}

\section{Metal free ceramic finished and characterized by manual polishing}

\author{
Fernando Grandon ${ }^{1 *}$, Blas Galdames ${ }^{1}$, Natalia Marcus ${ }^{1}$, Maria Muster $^{2}$
}

\begin{abstract}
1. Departamento de Odontología Restauradora, Universidad de Concepción, Chile.

2. Departamento de Odontología Conservadora, Universidad Andrés Bello, Chile.
\end{abstract}

* Correspondencia autor: Fernando Grandón Villegas | Dirección postal: Facultad de Odontología Universidad de Concepción, Roosevelt 1550 Concepción, Chile. | Teléfono +56-41-2204481 | e-mail: fgrandon@udec.cl

Trabajo recibido el 03/10/2017. Aprobado para su publicación el 04/12/2017

\begin{abstract}
RESUMEN
La rehabilitación oral mediante prótesis libres de metal ha dado solución funcional y estética a diversas situaciones clínicas, sin embargo, coronas unitarias anteriores aún son consideradas un gran desafío estético. En ellas, al igual que en estructuras métalo-cerámicas, brillo y lisura superficial se obtienen comúnmente mediante glaseado. Esta técnica puede presentar algunas limitaciones para replicar características de superficie, especialmente en dientes con gran riqueza microanatómica. Caso: El objetivo es presentar la rehabilitación anterior unitaria mediante corona IPS E.max® terminada mediante pulido manual, logrando así ajustes clínicos, personalización microanatómica y una superficie suave y brillante, culminando con un resultado estético muy satisfactorio. Conclusiones: El acabado manual es un procedimiento ampliamente documentado, de fácil ejecución y bajo costo. Podría disminuir envíos repetidos al laboratorio protésico y así los efectos asociados a la sobrecocción cerámica, sin embargo, requiere conocimiento del tipo de cerámica a pulir y una aplicación sistemática.
\end{abstract}

PALABRAS CLAVE

Cerámicas dentales, Técnicas de pulido, Biomimética, Libre de metal.

Rev. Clin. Periodoncia Implantol. Rehabil. Oral Vol. 11(1); 39-42, 2018.

\begin{abstract}
Oral rehabilitation through metal free prosthetics has given functional and aesthetic solutions to various clinical conditions; nevertheless, single-tooth rehabilitation in the anterior region is still considered a major challenge. In these crowns as well as in metallo-ceramic structures, the finishing is obtained by the glazing technique. This technique could show some limitations to replicate the surface characteristics, especially in teeth with a rich micro-anatomy. Case Report: The aim is to present an anterior single-tooth rehabilitation with IPS E.max® crown, and its finishing by hand polishing technique, achieving clinical adjustments and customized surface anatomy, a smooth surface, excellent brightness and a very satisfactory aesthetic result. Conclusions: Manual finishing technique is well documented, easy to execute and with low cost. Eventually, it could decrease repeated laboratory shipments and so the associated effects of the over-cooking of ceramic; nevertheless it requires knowledge of the type of ceramic to polish and a systematic clinical protocol implementation. KEY WORDS

Dental ceramics, Polishing methods, Biomimetic, Metal free.
\end{abstract}

Rev. Clin. Periodoncia Implantol. Rehabil. Oral Vol. 11(1); 39-42, 2018.

\section{INTRODUCCIÓN}

Los tratamientos rehabilitadores en el sector anterior requieren de materiales altamente predecibles y capaces de emular las características ópticas de las estructuras a reemplazar. Es por esto que las restauraciones libres de metal han ganado un importante espacio en el tratamiento de pacientes con elevadas demandas estéticas, abriendo un abanico de nuevas opciones que facilitan una integración biomimética y mayor conservación de la estructura dentaria sana ${ }^{(1)}$. Al enfrentarse a dientes tratados endodónticamente, especialmente aquellos que evidencian cracks o fisuras, la evidencia se inclina por restauraciones de recubrimiento completo, pudiendo éstas disminuir el riesgo de fracturas catastróficas ${ }^{(2)}$. Además, los tratamientos en el sector anterior requieren una planificación y técnica depurada, siendo la restauración de un incisivo central una instancia sumamente desafiante, que necesita una estrecha colaboración entre odontólogo y técnico protesista. El desarrollo de sistemas cerámicos libres de metal ha generado un arsenal de nuevas alternativas, que equilibran factores estéticos, biológicos y mecánicos. A pesar de esto, dichos materiales pueden fracasar en el logro de una biomimética satisfactoria, influenciada fuertemente por aspectos como translucidez y textura superficial(3).

En relación a la terapia de blanqueamiento en muñones decolorados, Tam y cols. ${ }^{(4)}$ proponen evitarla, por el eventual efecto negativo en las características microestructurales de la dentina a través del contacto directo con agentes clareadores por periodos extensos. Tratamientos de clareamiento domiciliario, en cambio, resultan efectivos y con baja 
tendencia a recidivar, lo que permitiría su aplicación con seguridad como complemento de tratamientos rehabilitadores.

Independiente de la elección de un sistema métalo-cerámico tradicional o un sistema libre de metal, el protocolo común de terminación sigue siendo la técnica de glaseado, la cual deja una superficie suave y con alto brillo superficial(5), sin embargo, su aplicación puede impactar negativamente en la mantención de la microanatomía superficial en dientes muy texturizados. La remoción de la capa de glaseado puede ser necesaria durante los ajustes clínicos asociados a relación oclusal y morfología, pero la superficie cerámica puede ser fácilmente pulida mediante el uso de elementos diseñados especialmente para este fin ${ }^{(6)}$. Yilmaz y col. ${ }^{(7)}$ postulan que se deben evitar cocciones repetidas de la cerámica, ya que esto tiene efectos destructivos en la capa superficial de la cerámica.

Las dudas generadas hasta ahora sobre el potencial abrasivo de la cerámica pulida en los dientes antagonistas, la eventual retención de biofilm ${ }^{(8)}$ y su menor resistencia han sido desestimadas con los aportes de evidencia reciente ${ }^{(9)}$. Hoy es sabido que el desgaste de estructuras antagonistas es similar y en algunos casos, incluso menor cuando la cerámica es terminada mediante pulido manual, al compararla con cerámica glaseada ${ }^{(3,10)}$.

Aspectos como suavidad superficial y brillo pueden ser completamente restablecidos cuando se aplica el protocolo de pulido correcto al tipo de cerámica que se está utilizando(11). Esto ha generado nuevas posibilidades para el clínico y el protesista dental, pues de esta forma se puede evitar envíos al laboratorio, con el consiguiente ahorro de tiempo, evitando así cocciones repetidas. Así también, en situaciones donde el patrón de textura superficial es complejo y difícil de reproducir, permite caracterizar la superficie manualmente, con observación directa de los dientes naturales, lo que sin duda contribuye a localizar de mejor manera las áreas de reflexión de luz, logrando así un resultado más natural.

Este reporte tiene por objetivo presentar la resolución de una discromía en un diente fisurado y endodónticamente tratado mediante la confección de una corona cerámica libre de metal, exponiendo la técnica de pulido mecánico como una herramienta válida para personalizar y generar un excelente acabado en superficies cerámicas, especialmente en dientes jóvenes con gran textura superficial, difíciles de reproducir con el glaseado tradicional.

\section{REPORTE DE CASO}

Paciente sexo femenino, 17 años de edad, sin antecedentes sistémicos relevantes, consulta por estética deficiente de la sonrisa, y en especial, por el oscurecimiento de su incisivo central izquierdo. La paciente había recibido tratamiento de endodoncia del diente 2.1 y posterior terapia de blanqueamiento interno por decoloración posterior, terapia que no generó los resultados por ella esperados.

Durante la evaluación de la sonrisa se observa armonía en la línea incisal, buen volumen labial y decoloración del diente 2.1, a la vez que se aprecia una fisura en la cara vestibular del mismo y gran riqueza microanatómica. Al examen radiográfico se observan incisivos centrales con longitud radicular disminuida. Clínicamente se aprecia ausencia de movilidad dentaria y de lesiones de caries activas, buena alineación dentaria, pero con algunos signos de higiene bucal deficiente, además de gran pérdida de estructura en el diente 2.1 por acceso endodóntico.

Después de reforzar los hábitos de higiene, se planifica el tratamiento rehabilitador, el que se inició con clareamiento domiciliario utilizando Opalescence ${ }^{\circledR}$ PF $10 \%$ (Ultradent Products Inc., South Jordan, UT, EEUU.), bajo modalidad de aplicación diurna por cuatro horas diarias durante dos semanas, excluyendo de la terapia al diente 2.1 en el cual se planifica una corona periférica unitaria (PFU) totalmente cerámica sobre muñón mixto con inserción de fibroposte. Se optó por una restauración de recubrimiento completo considerando el fracaso del clareamiento intracameral antes realizado, sumado a la presencia de una fractura incompleta vestibular corriendo en dirección cérvico incisal (Fig. 1), lo que constituye una situación de alto riesgo de fractura catastrófica. Luego se procedió a la cementación del poste de fibra de cuarzo DT. Light Post Illusion ${ }^{\circledR}$ (RTD, Grenoble, France) $n^{\circ} 4$ con cemento de resina autograbante Smartcem2 ${ }^{\circledR}$ Light (Dentsply DeTrey, Konstanz, Germany), polimerizado con lámpara Led a una potencia de $3200 \mathrm{~mW} / \mathrm{cm}^{2}$ (Valo, Ultradent Products Inc., South Jordan, UT, EEUU). Antes de realizar la preparación dentaria se tomó una matriz de silicona pesada para confeccionar el provisional.

En consideración a la alta demanda estética del caso y el patrón de superficie de los dientes 1.1 y 2.1 (Fig. 2), se elige como material restaurador el sistema cerámico IPS E.max ${ }^{\circledR}$ (Ivoclar Vivadent, Schaan, Liechtenstein), que implicó la confección de un casquete cerámico inyectado en base a disilicato de litio, más la aplicación posterior de masas de cerámica feldespática con nanoflúorapatita como cerámica de recubrimiento.

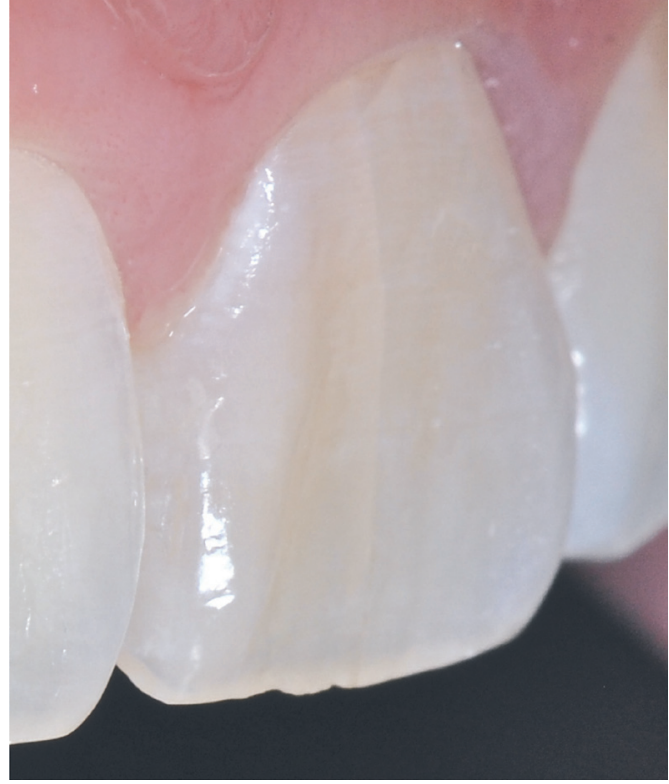

Figura 1. Fractura incompleta vestibular en dirección cérvico-incisal

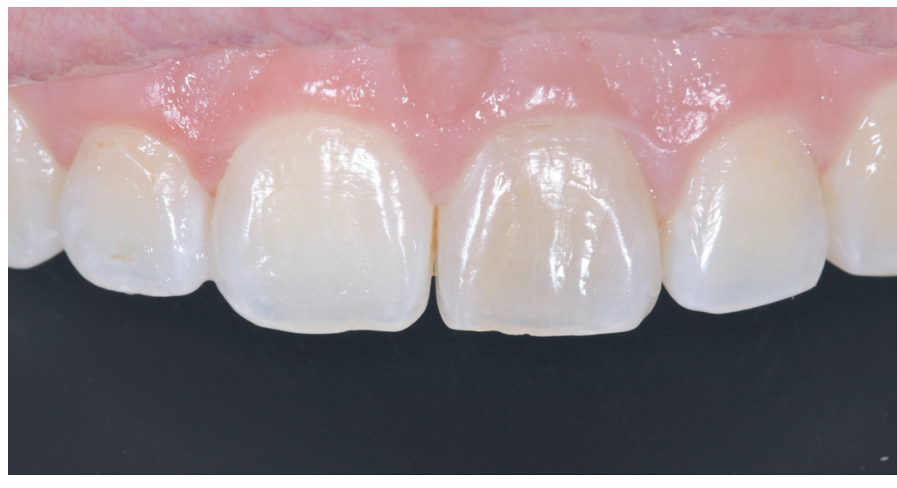

Figura 2. Patrón de superficie de incisivos centrales

Luego de la confección del provisional y estabilización del color post clareamiento, se procedió a la toma de impresión con silicona por adición Panasil Putty e Initial Contact Light (Kettenbach GmbH \& Co.KG, Eschenburg, Germany). Un aspecto importante de este procedimiento es garantizar volúmenes que permitan una adecuada resistencia y enmascaramiento del muñón oscurecido, lo que se puede evaluar fácilmente al superponer la matriz en sentido frontal e incisal.

En sistemas cerámicos más translúcidos, el color es producto de la interacción óptica entre el sustrato dentario, el material restaurador y el agente de fijación. Por esto, es necesario protocolizar con el técnico la toma de color, la que además debe considerar el color del sustrato o color de partida, utilizando para esto el colorímetro de muñones Natural Die Material ${ }^{\circledR}$ (Ivoclar Vivadent, Schaan, Liechtenstein), registrándose un color ND5 (Fig. 3a).

El color final que se deseaba obtener luego de la aplicación se aproximaba al color $1 \mathrm{M} 1$ del colorímetro 3D Master $^{\circledR}$ (Vita Zahnfabrik, Bad Sackingen, Germany), luego de una terapia de clareamiento exitosa en los dientes vecinos (Fig. 3b). Entendiendo la dinámica del sistema cerámico seleccionado, se decidió enmascarar la decoloración del muñón con un adecuado manejo de los espacios y el material de fijación. No obstante, la textura superficial, compleja y rica en elementos verticales y horizontales se hizo dificultosa de replicar para el técnico protesista, lo que concluyó con una corona bien lograda en términos generales, pero con una apariencia distinta al central homólogo, esto debido principalmente a pequeñas diferencias en el ancho mesio-distal sumado a discrepancias en la reflexión de la luz, elemento muy influyente en la percepción final del color (Fig. 4). Esta situación, analizada bajo la perspectiva tradicional, amerita un nuevo envío al laboratorio dental con el fin de realizar un reglaseado de la superficie e introducción en el horno cerámico a altas temperaturas. Este procedimiento trae asociado varios inconvenientes, como aumentar los tiempos de tratamiento y eventualmente sus costos, cambios de color en la cerámica por sobre cocción y posibilidad de no replicar el patrón de superficie requerido. Es 

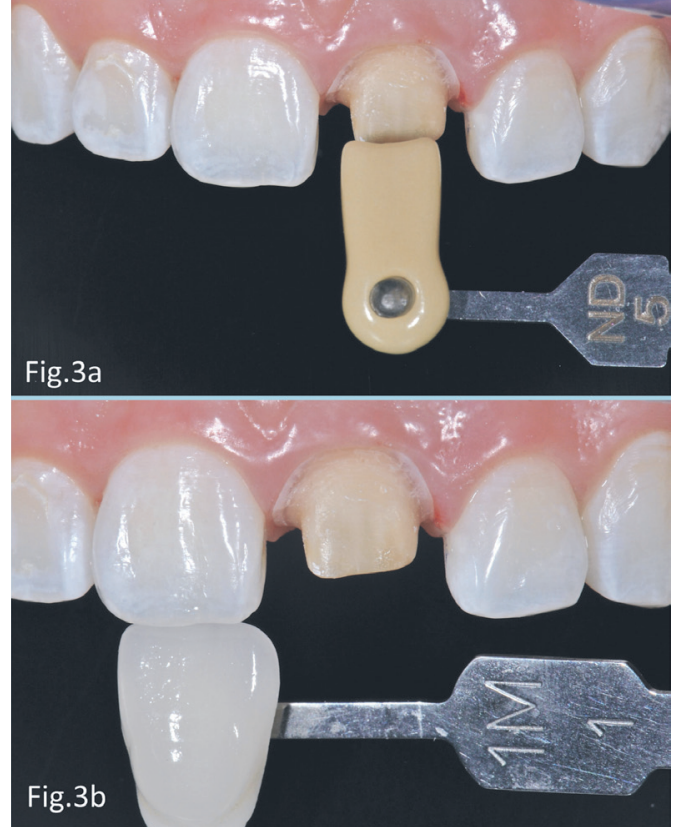

Figura 3a. Registro de color de de muñón

Figura 3 b. Registro de color dentario

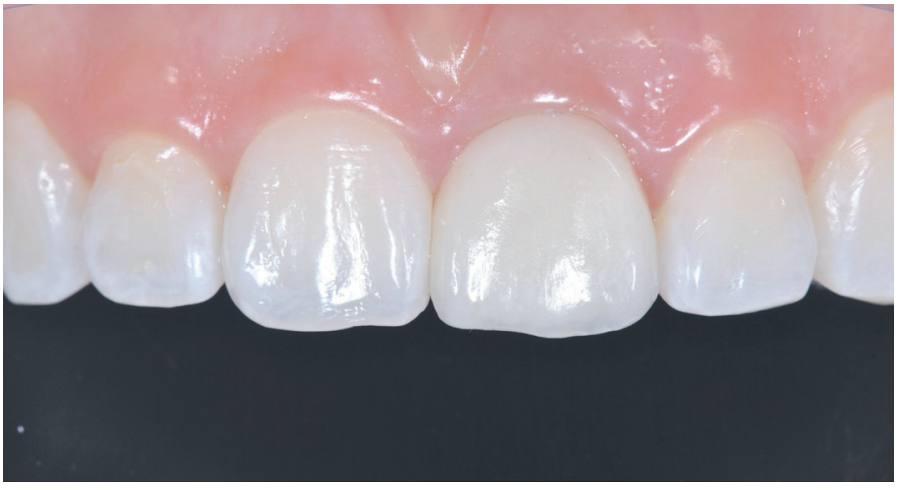

Figura 4. Prueba cerámcia IPS E.max ${ }^{\circledR}$ glaseada

por esto que, en contraposición, se realizó un retallado de la superficie con fresas diamantadas de grano fino hasta obtener una réplica de la anatomía superficial del diente vecino. Luego se procedió al pulido manual utilizando el sistema Optrafine ${ }^{\circledR}$ (Ivoclar Vivadent, Schaan, Liechtenstein), comenzando primero por el pulidor más abrasivo (Fig. $5 a)$, para luego continuar con el pulidor de grano más fino (Fig. 5b). La secuencia culminó con la aplicación de una pasta de pulido mediante escobilla, también incluida en el sistema (Fig. 6)

Luego de la caracterización y pulido manual de la cara vestibular se procedió a la cementación adhesiva de la corona, que incluyó su acondicionamiento siguiendo los protocolos descritos para este tipo de sistemas cerámicos. Para el sustrato dentario se aplicó el sistema adhesivo Single Bond Universal ${ }^{\circledR}$ (3M ESPE, Neuss, Germany.), combinado al agente cementante de polimerización dual RelyX Ultimate ${ }^{\circledR}$ color A1 (3M ESPE, St.Paul, MN, EE.UU.). Luego de su fijación se obtuvo una prótesis unitaria libre de metal integrada a dientes vecinos y antagonistas (Fig. 7) y con resultados estéticos muy satisfactorios (Fig. 8a,b).

En los controles posteriores, a los 6, 12, 18 y 24 meses no se han observado tinciones ni desgaste de la cerámica, además de mantención de salud de los tejidos blandos, por lo que se considera exitoso el tratamiento. Se sugieren controles posteriores para asegurar el correcto mantenimiento de la PFU libre de metal.

\section{DISCUSIÓN}

Durante las últimas décadas, las cerámicas dentales han sido ampliamente utilizadas con fines protésicos, dada su alta biocompatibilidad y excelentes propiedades estéticas. El advenimiento de nuevas tecnologías para su confección y fijación ha permitido conseguir resultados cada vez más naturales, ampliando sus aplicaciones en

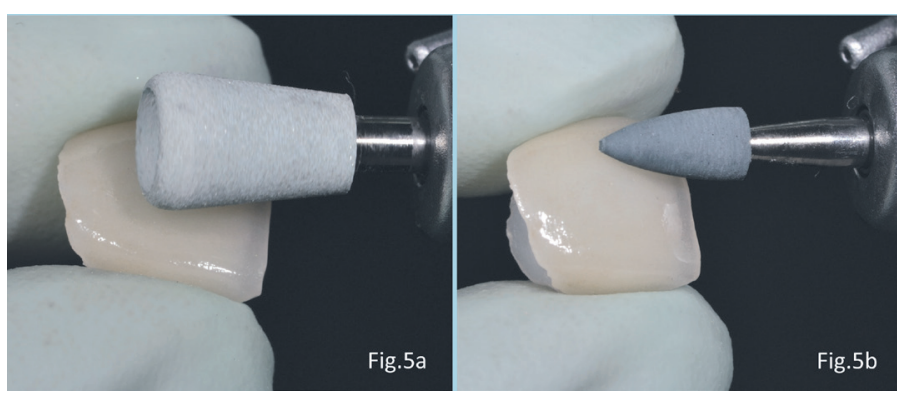

Figura 5a. Pulido manual con sistema Optrafine ${ }^{\circledR}$. Pulidor abrasivo

Figura 5b. Pulido manual con sistema Optrafine ${ }^{\circledR}$. Pulidor fino

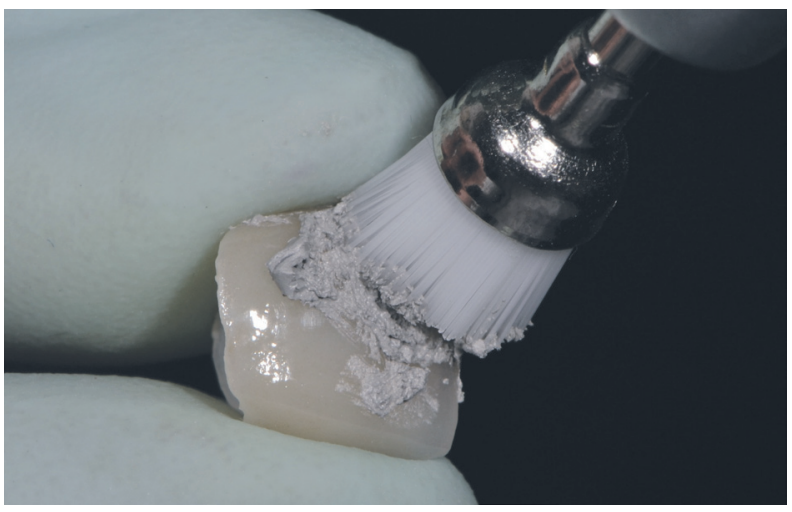

Figura 6. Finalización secuencia de pulido manual con pasta de pulido Optrafine ${ }^{\circledR}$

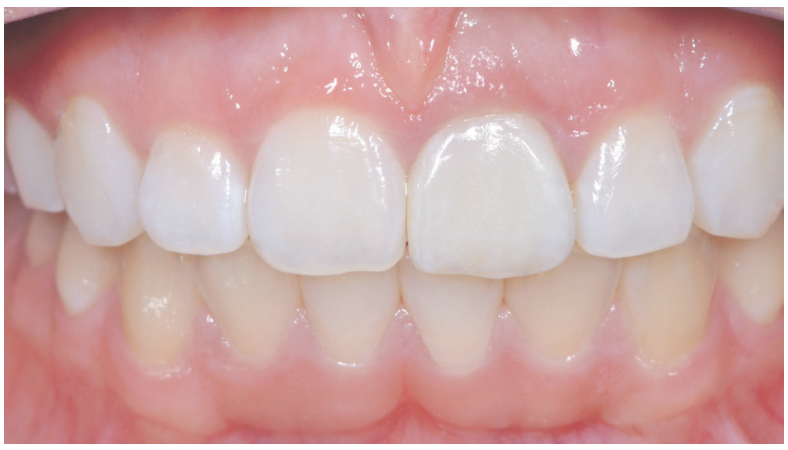

Figura 7. Prótesis unitaria libre de metal integrada a dientes vecinos y antagonistas
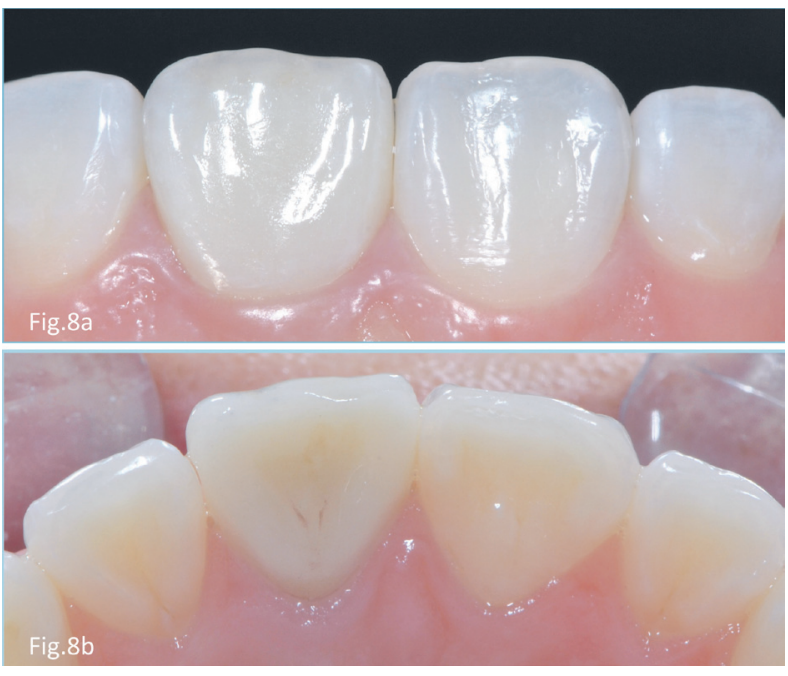

Figura 8a. Rehabilitación anterior pieza 2.1 mediante cerámica IPS E.Max ${ }^{\circledR}$ pulida de forma

Figura $\mathbf{8 b}$. Vista palatina 
implantología y odontología restauradora.

La restitución de la suavidad y brillo superficial mediante pulido mecánico como alternativa al glaseado adquiere validez, pues no sólo permite lograr personalización de la anatomía superficial, como el caso desarrollado, sino que constituye también una herramienta útil en situaciones comunes como el ajuste clínico de contactos proximales, corrección de contornos inadecuados y ajuste oclusal. Consecuencia de ello, la elevada rugosidad superficial de la estructura cerámica se ha asociado a retención de biofilm, pigmentación, estética insatisfactoria y disminución de la resistencia debido a la propagación de fisuras y defectos $^{(11)}$

Por otra parte, es sabido que cocciones repetidas de la cerámica pueden llevar a un efecto negativo en el color, porosidad, resistencia y ajuste marginal sobre distintas subestructuras ${ }^{(7,12)}$. La potencial influencia negativa de cocciones repetidas se confirma con el estudio in vitro de Zeighami y cols. ${ }^{(13)}$ en el que expone una disminución de la resistencia al test de microtensión entre cerámica de recubrimiento y subestructuras de circonio, lo que realza las ventajas de evitar nuevas cocciones.

Los sistemas disponibles para el pulido mecánico están representados por pulidores siliconados, piedras montadas de distinta granulación, discos diamantados flexibles, fieltros y escobillas, las que pueden incluir o no pastas abrasivas ${ }^{(10)}$. Aún no existe evidencia que relacione elementos como velocidad de pulido y presencia de agua como determinantes de la calidad del mismo, sin embargo la falta de consenso respecto a qué método o sistema de pulido elegir habitualmente conlleva a confusiones ${ }^{(11)}$.

Para obtener resultados óptimos resulta indispensable conocer qué tipo de cerámica estamos puliendo, además de respetar todos los pasos que el fabricante recomienda ${ }^{(14)}$. Los autores de este trabajo se inclinan por sistemas de pocos pasos, además que permitan ajuste extraoral y ajuste intraoral, siendo por esto deseable que se puedan acoplar a contraángulo, tal como el sistema Optrafine ${ }^{\circledR}$, siendo común la necesidad de ajuste después de la cementación. Muchos sistemas cerámicos están constituidos por cerámicas de distinta composición en la subestructura y en la cerámica de recubrimiento. Este es el caso del sistema IPS E.max ${ }^{\circledR}$, por lo cual en la selección del sistema más apropiado para su pulido, se consideró que el fabricante asegurara su efectividad en la cerámica feldespática de recubrimiento ${ }^{(15)}$. Atención merecen también la técnicas de cerámicas maquilladas, puesto que estas técnicas impiden la aplicación de protocolos de pulido por la eventual eliminación del maquillaje superficial de forma parcial o total, recomendándose en estos casos aplicación del glaseado tradicional como método de terminación ${ }^{(14)}$.

\section{CONCLUSIÓN}

La técnica de pulido mecánico aplicada posterior a la etapa laboratorial, permitiría personalizar la superficie y generar un excelente acabado y patrón de brillo, sin embargo, se debe aplicar un protocolo sistemático, acorde al tipo de cerámica que se pretende pulir. Es un procedimiento simple, de bajo costo y eventualmente, ayudaría a optimizar los tiempos clínicos.

\section{CONFLICTO DE INTERÉS}

Los autores relatan no tener conflictos de interés.

\section{Bibliografía}

1. Dunn D, Walters BD. Predictable aesthetic excellence with all ceramic restorations - a new paradigm for success. Aust Dent Pract. 2012;23(2):128-38.

2. De Castro MAM, Poi WR, de Castro JCM, Panzarini SR, Sonoda CK, Trevisan $\mathrm{CL}$, et al. Crown and crown-root fractures: an evaluation of the treatment plans for management proposed by 154 specialists in restorative dentistry. Dent Traumatol. 2010 Jun;26(3):236-42.

3. Akar GC, Pekkan G, Cal E, Eskitaşçığlu G, Ozcan M. Effects of surface-finishing protocols on the roughness, color change, and translucency of different ceramic systems. J Prosthet Dent. 2014 Mar 8;112(2):313-21.

4. Tam LE, Kuo VY, Noroozi A. Effect of prolonged direct and indirect peroxide bleaching on fracture toughness of human dentin. J Esthet Restor Dent. 2007 Jan;19(2):100-9; discussion 110.

5. Magne P, WS O, Pintado M, DeLong R. Wear of enamel and veneering ceramics after laboratory and chairside finishing procedures. J Prosthet Dent. 1999;82(6):669-79.

6. Jagger D, Harrison A. An in vitro investiga- tion into the wear effects of unglazed, glazed and polished porcelain on human enamel. J Prosthet Dent. 1994;72:320-3.

7. Yilmaz K, Ozkan P. Profilometer evaluation of the effect of various polishing methods on the surface roughness in dental ceramics of different structures subjected to repeated firings. Quintessence Int. 2010;41(7):e125-31.

8. Bollen CM, Lambrechts P, Quirynen M. Comparison of surface roughness of ora hard materials to the threshold surface roughness for bacterial plaque retention: a review of the literature. Dent Mater. 1997 Jul;13(4):258-69.
9. Heintze SD, Cavalleri a, Forjanic M, Zellweger G, Rousson V. Wear of ceramic and antagonist--a systematic evaluation of influencing factors in vitro. Dent Mater. 2008 Apr;24(4):433-49.

10. Yuzugullu B, Celik C, Erkut S, Ozcelik TB. The effects of extraoral porcelain polishing sequences on surface roughness and color of feldspathic porcelain. Int $J$ Prosthodont. 2009;22(5):472-5.

11. Da Silva TM, Salvia ACRD, de Carvalho RF, Pagani C, da Rocha DM, da Silva EG. Polishing for glass ceramics: Which protocol? J Prosthodont Res. 2014 Mar 28;58(3):160-70.

12. Komine $F$, Shiratsuchi $H$, Kakehashi $Y$, Matsumura $H$. Influence of porcelain-firing procedures on the marginal distortion of electroformed metal-ceramic restorations. Quintessence Int. 2008;38(10):E583-8.

13. Zeighami $S$, Mahgoli $H$, Farid F, Azari A. The effect of multiple firings on microtensile bond strength of core-veneer zirconia-based all-ceramic restorations. J Prosthodont. 2013 Jan;22(1):49-53.

14. Yilmaz K, Ozkan $P$. The methods for the generation of smoothness in dental ceramics. Compend Contin Educ Dent. 2010;31(1):30-2, 34, 36-8 passim; quiz 42, 44.

15. Capelozza J, Nishida R, Afif A, Martins D, Santos J, Alves E et al. Effect finishing and polishing procedures on the surface roughness of IPS Empress 2 ceramic. Acta Odontol Scand. 2013;71(3-4):438-43. 